\title{
Planck Speed: the Missing Speed of Physics? Absolute Still Without Breaking Lorentz Symmetry!
}

\author{
Espen A. Haug
}

\begin{abstract}
Today, the speed of light is one of the cornerstones of modern physics. It is the maximum speed limit for transferring information, and plays a very central role in relativity theory. It also seems to be the same as the assumed and measured speed of gravity (gravitons and/or gravitational waves). We are not questioning the speed of light, as we indeed think it is the maximum speed limit and that it is identical to the speed of gravity, but we also think photons lead us to a second speed limit, which we will term the Planck speed. Photon-photon collisions are part of modern physics, but what is not discussed is the speed of two photons during the very moment of a photon-photon collision. How can photons move during a collision? Or, assume a photon is reflected by a mirror; to do so it must collide with the building blocks of the mirror, yet how can it be moving during the very collision point? Also, modern physics assumes mass can be created in a photon-photon collision. We suggest this mass is the Planck mass particle and that it only lasts one Planck time, so when two photons collide, we will claim they must stand still for the direct observer. That is, we suggest that the speed of the Planck mass particle is always zero, but that this zero-velocity can only last for one Planck time. That it only lasts one Planck time means it will not break with the relativity principle or Lorenz symmetry, or at least what we can call weak Lorentz symmetry. We will show that the missing Plank mass particle is the very collision point between two photons, and that this is fully in line with predictions in other researchers' findings in relation to the possible photon mass. This new view seems to solve the missing mass-gap, and seems able to unify gravity with quantum mechanics. This again seems to unify photons with mass, because one of the long-outstanding questions in physics is whether photons have mass or not. In addition, we show how to find, in a very simple way directly from gravitational observations, $\frac{l_{p}}{t_{p}}=c_{g}=c$, which is the upper speed limit (the speed of light and gravity) independent of any knowledge of $G$ or $\hbar$ or $c$.
\end{abstract}

\section{Index Terms}

Planck scale, Planck mass particle, photon-photon collision, Compton wavelength, Planck length, Planck speed, speed of gravity.

\section{Short introduction to Planck Units AND HOW THEY ARE ASSUMED TO BE MAXIMUM AND MINIMUM LiMITS}

Assuming there were three very important universal constants, namely the speed of light, the Newton gravitational constant, and the Planck constant, and then combining these with dimensional analysis, Max Planck [1] in 1899 derived a unique length $l_{p}=\sqrt{\frac{G \hbar}{c^{3}}}$, a time $t_{p}=\sqrt{\frac{G \hbar}{c^{5}}}$, a mass $m_{p}=\sqrt{\frac{\hbar c}{G}}$, and a temperature (energy) $T_{p}=\sqrt{\frac{\hbar c^{5}}{G k_{B}^{2}}}$.

Eddington [2] was likely the first to suggest the Planck length could be linked to quantum gravity. Today many, if not most, physicists, in particular those working with quantum gravity theory, assume the shortest possible observable length is the Planck length; see, for example [3], [4], [5], [6]. Still, there are other physicists who have questioned even the existence of Planck length since it is assumed it can only be derived from dimensional analysis; see Unzizker [7]. However, recently, it has been shown how the Planck length can be extracted from gravity observations independent on any knowledge of $G$ or $\hbar$; see Haug [8], [9], [10], [11], [12]. We [13], [14], [15] have recently also formulated a quantum gravity theory in which all observable gravity phenomena are only dependent on the two constants, namely $l_{p}$ and $c$. We mention this as it supports the idea that the Planck length is something very special and indeed likely linked to an absolute minimum length and minimum time. This strongly supports the importance of the Planck scale, and the contention that Planck units should play a central role in a unified quantum gravity theory.

Gorelik and Ozernoi [16] suggest that the maximum possible temperature is the Planck temperature. Caianiello [17], in 1981, was perhaps the first to suggest there is likely a maximum acceleration. In 1984, Scarpetta [18] was the first to introduce what he called the Planck acceleration, which he suggested is this maximum acceleration (see also [19], [20]). That the Planck acceleration is the maximum acceleration is a view also held by, for example, Landsberg and Falle [21]. For an overview of more Planck units, see [22]. An important point here is that most, if not all, the Planck units seem to be linked to either minimum limits or maximum limits. The Planck length is likely the minimum length, the Planck time is the minimum time, the Planck mass is likely the maximum possible mass for an elementary particle, the Planck acceleration is the maximum acceleration, the Planck temperature is the maximum temperature, and the Planck energy is the maximum energy etc. The maximum speed of anything carrying information is known to be the speed of light. Could there also be a minimum speed linked to the Planck units? We think so and it is, in our view, directly linked to the Planck mass particle and all the Planck units. 


\section{The Planck SPEED}

The speed of light ${ }^{1}$ is constant and the same from any reference frame. More precisely, the round-trip speed of light has been confirmed to be constant and isotropic as observed from any reference frame. On the other hand, to measure the one-way speed of light is maybe impossible. This is because to measure the one-way speed of light would generally require the use of two synchronized clocks placed a distance apart, and to synchronize them one would generally need to know the one-way speed of light, as first pointed out by Poincare [23]. Poincare therefore suggested that for synchronization purposes one could assume the one-way speed of light is the same as the round-trip speed of light. Einstein went one step further and abandoned the ether since, to detect the ether, one generally has to be able to measure the one-way speed of light. No matter the view on the one-way speed of light, it will (at least in general) be isotropic and constant as measured with Einstein's synchronized clocks. When it comes to rotating frames, it is much more complex; see, for example [24], [25], [26], [27], but rotating frames are outside the scope of this paper.

The speed of light also gives the maximum velocity of any signal that can carry information. Also, gravity has been confirmed to be traveling at the speed of light or at least very close to the speed of light; for example, Abbott et. al. [28] has been able to determine the difference between the speed of gravity and the speed of light as being maximum only between $-3 \times 10^{-15}$ and $+7 \times 10^{-16}$ times the speed of light; see also [29], [30], [31], [32], [10].

We fully agree that the speed of light is $c$ and also that the speed of gravity is $c_{g}=c$. However, we are asking: What is the speed of a photon in the moment it is reflected, or hits something like a mirror? What is the speed of a photon in the very moment it collides with another photon, in a so-called photon-photon collision? What is well known is that photon-photon collisions can create mass[33].

The speed of photons during a photon collision, or during the moment of reflection in a "perfect mirror" is, to our knowledge, not much discussed in standard physics. One could try to use quantum mechanics, but we have reason to think standard quantum mechanics break down at the point where the Planck scale enters the equation. Recent research that we have published in relation to a unified quantum gravity theory indicates that the speed of photons in a collision is, strangely enough, always zero. We have also demonstrated how two colliding photons have a mass equal to the Planck mass; they are, so to speak, creating the Planck mass. This mass only lasts the Planck time. First of all, it is important to be aware that any mass in kilograms can be expressed as:

$$
m=\frac{\hbar}{\bar{\lambda}} \frac{1}{c}
$$

where $\bar{\lambda}$ is the reduced Compton wavelength. This way of expressing the mass, even if trivial was possibly first described by Haug [34] in 2018. This is actually nothing more than the reduced Compton [35] wavelength formula, $\bar{\lambda}=\frac{\hbar}{m c}$ solved with respect to $m$. For a relativistic mass we must have:

$$
m_{r}=m \gamma=\frac{\hbar}{\bar{\lambda}} \frac{1}{c} \gamma=\frac{\hbar}{\bar{\lambda} \sqrt{1-\frac{v^{2}}{c^{2}}}} \frac{1}{c}
$$

Bear in mind that it was Lorentz [36] in 1899 who first suggested the relativistic mass of the form $m \gamma$. Einstein [37] at the end of his 1905 paper suggested two relativistic masses, namely $m \gamma^{2}$ and $m \gamma^{3}$, which are both incorrect according to what is today considered relativistic mass; see, for example [38], [39]. With the invention of Minkowski's [40] geometry for space-time and the four vector approach, Einstein abandoned relativistic mass altogether; see [41]. General relativity theory therefore totally ignores relativistic mass; something that has recently been pointed out could be a weakness in the theory; see [42], [43]. Einstein relied on relativistic momentum instead. The relativistic momentum that was first described by Max Planck [44] in 1906 was later also used by Einstein. Several researchers claimed the relativistic momentum is consistent with relativistic mass, something which agrees with our line of thought. That is, the relativistic mass is changing when the mass is moving or, more precisely, there is a length contraction in the reduced Compton wavelength of the mass. On the other hand, Einstein, as well as researchers like Hecht [41] and Adler [45], claimed the mass is always the rest-mass. Let's look at the relativistic momentum formula:

$$
p=m v \gamma=\frac{m v}{\sqrt{1-\frac{v^{2}}{c^{2}}}}
$$

According to the view that have abandoned relativistic mass this would mean $m$ is not affected by the motion even as observed from the other frame, here $m$ is always the rest-mass. For a given rest-mass $m$, this mass is then a constant. It must then be $v$ that is relativistic and of the form:

$$
v \gamma=\frac{v}{\sqrt{1-\frac{v^{2}}{c^{2}}}}
$$

This relativistic velocity would then be above $c$ when $v$ is above $\frac{c}{\sqrt{2}}$, which is an impossibility. All the relativistic momentum must come from changes in the mass; that is, from relativistic mass. Hecht [41] and others have an explanation to give if this

${ }^{1}$ At least as measured by Einstein's synchronized clocks 
is not the case? Is the relativistic momentum caused by some additional energy traveling with the mass? If so, where is this in the relativistic momentum formula? It is not there. Ignoring relativistic mass is likely one of the biggest blunders in modern physics, if not the biggest. To bring back relativistic mass in physics and also in gravity theory means we get a perfect match to supernova data without the invention of hypothetical dark energy. This has been demonstrated by Haug [46]. Further, one gets a much better fit with the Planck scale; see [42] and [43] as well as the Hubble scale. Absurd hypotheses, such as wormholes, also become mathematically impossible; see [47]. So perhaps we should indeed not reject relativistic mass due to prejudice, and at least investigate the idea of relativistic mass more carefully before we make conclusions. Returning to the relativistic mass if, in addition, the shortest possible length is the Planck length, then we must conclude that also the relativistic length of the contracted Compton wavelength can never get shorter than the Planck length. That is, we must have:

$$
\bar{\lambda} \sqrt{1-\frac{v^{2}}{c^{2}}} \geq l_{p}
$$

where $\overline{\bar{\lambda}}$ is the reduced Compton wavelength of the particle in question. Solved with respect to $v$ this gives:

$$
v \leq c \sqrt{1-\frac{l_{p}^{2}}{\bar{\lambda}^{2}}}
$$

This means the maximum velocity for any elementary particle with rest-mass must be:

$$
v_{\max }=c \sqrt{1-\frac{l_{p}^{2}}{\bar{\lambda}^{2}}}
$$

This is the same maximum velocity for anything with rest-mass that we have presented in a series of published papers [48], [49], [8], [13]. We also presented a more generalized version of this maximum velocity formula on October 15, 2015 at the Royal Institution in London, based on my 2014 book [50]. However, back then, we did not know how to find the Planck length independent of $G$, something we were able to do later on; see [8], [9], [10], [11]. Also, pay attention to the fact that special relativity theory is not consistent with any type of minimum length. Any length, if observed from someone moving close enough to the speed of light, will be contracted to a length shorter than the Planck length (or any suggested minimum length); see [51] for a more in-depth discussion on this point. Also, how can the Planck length be the shortest length in a rest frame, as it will then be even shorter in another reference frame due to length contraction? Special relativity clearly seems inconsistent with a minimum length; however, there is a simple way around this.

The Planck mass, which in our view is related to the Planck mass particle that again is linked to the collisions between two photons, has, even for a rest-mass, a reduced Compton wavelength equal to the Planck length, so it has a maximum velocity equal to what we will coin the Planck speed, and it is given by:

$$
v_{p}=c \sqrt{1-\frac{l_{p}^{2}}{\bar{\lambda}^{2}}}=c \sqrt{1-\frac{l_{p}^{2}}{l_{p}^{2}}}=0
$$

The Planck speed can also be written as:

$$
v_{p}=c \sqrt{1-\frac{G m_{p}^{2}}{\hbar c}}=0
$$

In a photon-photon collision, we have demonstrated that this is likely the Planck mass particle. So, this means the Planck mass always stands still during its lifetime, which is only the Planck time. But how can this be? Doesn't this break with the relativity principle, because if someone travels relative to something that one frame claims to stand still, then this object or frame will clearly move relative to a frame traveling relative to this reference frame, when observed from that other reference frame.

Surprisingly enough, the Planck mass particle being always at rest during its lifetime is not in conflict with the relativity principle or Lorentz symmetry (as we first thought [13]). If the Planck mass particle consists of two indivisible particles (building blocks of light) colliding, and each has a diameter equal to the Planck length, then the radius of the Planck mass particle is the Planck length (as we have suggested in several papers). Assume the Planck mass particle is simply a collision between the ultimate building blocks of light. Assume further that the ultimate building blocks of light are such indivisible particles with diameters equal to the Planck length. These indivisible particles, when not colliding, are moving at the speed of light (and are then light). However, when colliding, they are standing still relative to the indivisible particles they are colliding with. It is when standing still that they are a Planck mass particle. This collision lasts only the Planck time. This means the Planck mass particle can only be observed directly from itself. Thus, because nothing can travel away from the Planck mass particle in less than the Planck time, it cannot be observed from outside itself (based on the assumption that the Planck time is the shortest possible time and the Planck length the shortest possible length), except indirectly through some effects this collision has created. The effect of the duration of the collision is gravity, as clearly described in a series of recent papers [13], 
[14], [15]. The Planck speed is therefore essential to unify gravity and quantum mechanics. Standard quantum mechanics, we will claim, breaks down at the Planck scale and therefore can say nothing about gravity or the Planck speed. However, our recent unified quantum gravity theory can fully describe this.

\section{MASS IS COLLIDING PHOTONS?}

We assume all masses consist of indivisible particles moving at the speed of light back and forth over the reduced Compton wavelength of the particle in question [13]. When the indivisible particles move at the speed of light, then they are the building blocks of photons. Only when colliding with each other and standing still for the duration of the Planck time are they what we consider mass. The indivisible particles themselves are massless; it is the very collision with each other that is the origin of what we call mass. This mass is the Planck mass, but it only has a duration of the Planck time. For example, the kilogram mass of the electron will then be:

$$
m_{e}=\frac{c}{\bar{\lambda}_{e}} m_{p} t_{p}=\frac{\hbar}{\bar{\lambda}_{e}} \frac{1}{c} \approx 9.1 \times 10^{-31} \mathrm{~kg}
$$

This is the well-known kilogram mass of the electron. That is, in this model, the indivisible particles making up the mass each move back and forth over the reduced Compton wavelength of the electron at the speed of light; so as to collide at every reduced Compton time $\frac{\bar{\lambda}}{c}$. The very collision itself, however, lasts only the Planck time.

Thus, there is a Planck mass event, $\frac{c}{\lambda_{e}} \approx 7.76 \times 10^{20}$ times per second. Interestingly, this reduced Compton frequency is also very close to the tremble motion (zitterbewegung) frequency suggested to play a role for electrons by Schörodinger [52] in 1903, which corresponds to twice of the reduced Compton frequency of the electron. The Planck mass events are simply a collision between the building blocks of two photons, the indivisible particles. Inside a proton, such collisions happen at the reduced Compton frequency of the proton. The interest in the Compton wavelength of the proton goes back to at least 1958 (see [53]), and has recently garnered increased interest [54]. A Planck mass event at the Compton frequency of the proton again gives the experimental well-known mass of the proton. However, in the kilogram mass of the electron or proton or any other mass, all the information about the Planck scale disappears. That is, the collision time equal to the Planck time cannot be extracted from the kilogram mass. There is no Planck length or Planck time left to be extracted in the standard kilogram mass definition. As we have shown in earlier papers, the kilogram mass only indirectly contains information about the Compton frequency in matter, and this is all that is needed when one also describes energy as just a frequency. However, then one does not have information about the duration of the Planck mass event, which is the Planck time. Further, it is the duration of this collision, that is the Planck time, that is essential for gravity (and gravitational energy). A more complete mass definition is therefore likely collision-time as we have described in a series of papers on a new quantum gravity theory [13], [15]. We have also shown that the Planck mass particle (the collision of two indivisibles), when observed over a second, only corresponds to a kilogram mass off $1.17 \times 10^{-51} \mathrm{~kg}$ which is very small, and has energy equal to $\hbar f=\hbar \times 1$ per second. This is, interestingly, very close to the also-hypothetical photon mass suggested by a series of other researchers; see, for example [55], [56]. To detect the Planck scale, one should look for a very low energy, not a large energy, at the time scales our measurements are operating in. The gravitational force is considered a very weak force and we have recently given a quantum gravity model in which gravity is directly linked to these Planck mass events. Therefore, we need a macroscopic portion of mass to really detect gravity, which is a detection of the Planck scale. Interestingly, the mass of a photon-photon collision (two indivisible) particles is also the well-known Planck mass $\approx 2.17 \times 10^{-8} \mathrm{~kg}$ when observed in just the Planck time. That is, the Planck mass particle (the collision of two photons) is observational time-dependent. This is hard to grasp if one is new to our theory, so one may need to refer to our previous papers for an in-depth discussion about this.

\section{There is Also A MAXIMUm SPEEd GIVEn By THE PlancK UNITS, AND IT IS THE SPEED OF GRAVITY}

It is well known that the speed of light is also given by simply dividing the Planck length by the Planck time; $c=\frac{l_{p}}{t_{p}}$ (see for example [57], [58]). This is no surprise, as it is well known that $t_{p}=\frac{l_{p}}{c}$ can easily be found from the formulas given by Max Planck since we have $l_{p}=\sqrt{\frac{G \hbar}{c^{3}}}$ and $t_{p}=\sqrt{\frac{G \hbar}{c^{5}}}$, so this result is mathematically simple. Still, the most trivial results could be of great importance for understanding the quantum world. Since we know that, in general, nothing can move faster than the speed of light ${ }^{2}$ this can be seen as the upper speed limit indicated by Planck units. The Planck units therefore give us both a lower (absolute zero) and upper speed limit $c$. However there is a "big if" here, for as long as the Planck length $l_{p}$ and the Planck time $t_{p}$ can only be found from the three universal constants $G, c$, and $\hbar$, then this means simply inputting $c$ to find $c$ from $l_{p} / t_{p}$, we just get out exactly what we were putting into the model in the first place. The physics community is, for this and other reasons, therefore still divided on whether the Planck units really play an important role in physics or not. In particular, people working with quantum gravity theory seem to think the Planck units are of great importance, but have not been able to prove so, despite much effort to come up with ideas and experiments to detect the Planck scale (even just indirectly). The status until recently was quite nicely summarized by Eschini [59] in 2007:

\footnotetext{
${ }^{2}$ As measured by Einstein's synchronized clocks.
} 
"the significance of Planck's natural units in a future physical theory of spacetime is only a plausible, yet by no means certain, assumption."

In recent years [9], [11], [10], [12], we will claim there has been a breakthrough in understanding the Planck units as it has been demonstrated that the Planck length and Planck time can clearly be found independent of any knowledge of $G$ and $\hbar$. Still, in these papers, even if one does not need to know $G$ and $\hbar$, it seems that one needs to know $c$ directly or indirectly to find the Planck length and the Planck time. In this paper, we will demonstrate that to find $\frac{l_{p}}{t_{p}}$, it remarkably looks like we can find this from gravitational observations with no knowledge of $G, c$ and $\hbar$; can, in fact, find it actually independent of knowledge of any physical constants altogether. After this has been done, we can also find $l_{p}$ and $t_{p}$ independently (separately) without knowledge of also $c$ in addition to without knowledge of $G$ and $\hbar$. This because we first are extracting the speed of gravity directly from gravity observations with no knowledge of any physical constants as soon demonstrated.

The Planck length can be found from the following formula given by Haug [10], see appendix for derivation:

$$
l_{p}=\frac{\sqrt{\bar{\lambda}_{M} R_{L} R_{h}\left(T_{h}^{2}-T_{L}^{2}\right)}}{\sqrt{2\left(R_{h} T_{h}^{2}-R_{L} T_{L}^{2}\right)}}
$$

where $T_{h}$ and $T_{L}$ are two clocks that first have been synchronized when standing side by side, then one of them is slowly moved up in a tower (slow clock synchronization transportation ${ }^{3}$ ). We are considering standard gravitational time dilation in this formula. In practice, one would in addition likely have to adjust for velocity time dilation, as the rotation speed is different at different altitudes, but that effect is over a short time period likely insignificant. To adjust for also velocity time dilation is simple, but not shown here. Further $\bar{\lambda}_{M}$ is the reduced Compton wavelength of the gravitational object, that is Earth if performed on Earth. This reduced Compton wavelength of any gravitational object we have demonstrated can be found independently on any knowledge of $G$ and $\hbar$, see [9].

Further, the Planck time is given by (as first shown in this paper):

$$
t_{p}=\frac{R_{h}\left(T_{h}^{2}-T_{L}^{2}\right)}{2\left(R_{h} T_{h}^{2}-R_{L} T_{L}^{2}\right)} \sqrt{\frac{\bar{\lambda}_{M}}{g_{L}}}
$$

where $g_{L}$ is the measured gravitational acceleration at $R_{L}$. This formula we arrived at by simply understanding we must always have: $c=\sqrt{\frac{g \bar{\lambda} R^{2}}{l_{p}^{2}}}$, so $t_{p}=l_{p} / \sqrt{\frac{g \bar{\lambda} R^{2}}{l_{p}^{2}}}$, to find the Planck time formula above (Eq. 12) we simply need to replace $l_{p}$ with our Planck length formula above into this formula.

Next, if we divide the Planck length by the Planck time we get:

$$
\frac{l_{p}}{t_{p}}=\frac{\sqrt{2 g_{L} R_{L}\left(R_{h} T_{h}^{2}-R_{L} T_{L}^{2}\right)}}{\sqrt{R_{h}\left(T_{h}^{2}-T_{L}^{2}\right)}}=c_{g}=c
$$

where $c_{g}$ is the speed of gravity. We have tested the formula for a wide set of input and, as expected, it gives very close values to the known value of $c_{g}=c$. Pay attention to how the reduced Compton wavelength of the gravity object, $\bar{\lambda}_{M}$, in the formulas for $l_{p}$ and $t_{p}$, cancel each out when dividing $l_{p}$ with $t_{p}$. This is important, as to find the reduced Compton wavelength we likely indirectly are dependent on knowing $c$, so if the Compton wavelength was needed in the formula, one could think that the speed of light that we get out from the formula came from there, but that is clearly not the case as the Compton wavelength of the gravitational object cancels out for $l_{p} / t_{p}$. Further, we do not need to know the Planck constant to find the reduced Compton wavelength as explained in [9], [10], but anyway this is not so important here as it cancels out in the division of $l_{p} / t_{p}$.

We end up with a formula (Eq. 13) that only contains easily and directly observables. It depends on no knowledge of the value of any physical constant. There is no input of $G$, $\hbar$ or $c$, and still we get out $c_{g} \approx 299792458 \mathrm{~m} / \mathrm{s}$, which here represents $\frac{l_{p}}{t_{p}}$ since we get it directly from gravity observation. It is remarkable that this gives us the speed of gravity, which is identical to the speed of light in a vacuum. This is in strong contrast to the still-common view in the physics' community that the Planck units can only be found through dimensional analysis and that the Planck scale cannot be detected. This is, in our view, an indirect detection of the Planck scale, for even Newtonian gravity is actually a "concealed" quantum gravity theory in our view; see [13]. This is fully in line with our new quantum gravity theory that shows that all observable gravity phenomena can be predicted from only two constants, $c$ (or $c_{g}$ ) and $l_{p}$, in addition to variables; in other words, all gravity can be also be described from $l_{p}$ and $t_{p}$ since $t_{p}$ is simply $l_{p}$ divided by $c$, see [14], [15].

The formulas above can be simplified somehow by instead using approximations that work very accurately in weak gravitational fields, such as on the Earth (but that not is accurate in strong gravitational fields). The Planck length is then given by: (see also [10])

$$
l_{p} \approx \frac{\sqrt{\lambda_{M} R_{L} R_{h}\left(T_{h}-T_{L}\right)}}{\sqrt{R_{h} T_{h}-R_{L} T_{L}}}
$$

${ }^{3}$ This gives, for practical purposes, the same synchronization as Einstein's synchronization with light, see for example [60], [50]. 
and the Planck time as first presented here is then given by

$$
t_{p} \approx \frac{R_{h}\left(T_{h}-T_{L}\right)}{R_{h} T_{h}-R_{L} T_{L}} \sqrt{\frac{\bar{\lambda}_{M}}{g_{L}}}
$$

where $g_{L}$ is the measured gravitational acceleration at $R_{L}$. Further $l_{p} / t_{p}$, must then be equal to

$$
\frac{l_{p}}{t_{p}} \approx \frac{\sqrt{g_{L} R_{L}\left(R_{h} T_{h}-R_{L} T_{L}\right)}}{\sqrt{R_{h}\left(T_{h}-T_{L}\right)}} \approx c_{g}=c
$$

We have demonstrated that the upper speed limit $\frac{l_{p}}{t_{p}}=c_{g}$ can be easily extracted directly from gravity with no knowledge off $G, c$ or $\hbar$. One could argue we need to know $c$ to do velocity time dilation corrections to our clocks, but still what we are extracting in the end is from gravitational observations, so the speed we get out must be directly linked to the speed of gravity and not just the speed of light. In our view, this is a much simpler way to detect the speed of gravity that also demonstrates that gravity actually is directly linked to $l_{p}$ and $t_{p}$. The Planck time is actually how long a duration an indivisible particle moves at the speed of light during the time two colliding indivisibles are at absolute rest. In other words, $\frac{l_{p}}{t_{p}}$ contains both how fast the indivisible particle moves when not colliding and how long the collision is at rest (speed zero), namely the Planck time. In other words, we do not need to rely on complex measures of gravitational waves [30] etc. to measure the speed of gravity.

Alternatively, to using two clocks, we can simply measure the gravitational redshift from a laser beam sent from a tower. In the weak gravitational field, the Planck length can now be found very accurately by the following formula: (see [10])

$$
l_{p} \approx \sqrt{\frac{z R_{L} R_{h} \bar{\lambda}_{M}}{R_{h}-R_{L}}}
$$

where $z$ is the gravitational redshift measured from a laser beam sent from $R_{h}$ to $R_{L}\left(R_{h}>R_{L}\right)$; for example, from a laser beam sent from a tower down towards the surface of the Earth. $R_{h}$ will then be the distance from the top of the tower to the centre of the Earth and $R_{L}$ the distance from the bottom of the tower to the centre of the Earth. Further, $\bar{\lambda}_{M}$ is as before the reduced Compton wavelength of the gravitational object (the Earth). Further, the Planck time is given very accurately in a weak gravitational field by (as first presented here):

$$
t_{p} \approx \frac{z R_{h}}{R_{h}-R_{L}} \sqrt{\frac{\bar{\lambda}_{M}}{g_{L}}}
$$

Dividing the Planck length by the Planck time we get:

$$
\frac{l_{p}}{t_{p}} \approx \sqrt{\frac{g_{L} R_{L}\left(R_{L}-R_{h}\right)}{z R_{h}}} \approx c_{g}=c
$$

Again, pay attention to the fact that the reduced Compton wavelength of the gravitational mass that we needed to find using $l_{p}$ and $t_{p}$ cancels out when we divide the Planck length by the Planck time. This is remarkable as we have clearly demonstrated the upper speed limit is also easily given by the ratio of the Planck length to the Planck time. This can be experimentally observed without any knowledge off $G, \hbar$, or $c$. This again demonstrates our new view that gravity is the Planck scale. Even Newton gravity is Planck scale gravity at a deeper level, so the speed of gravity in the Newton gravitational theory is also at the deepest level the speed of light, and not infinite as thought by modern physics; see [10] for an in-depth discussion of this.

\section{CONCLUSion}

The Planck speed is the important missing speed in physics, and plays a very important role in understanding the link between the quantum world and the atomic as well as the macroscopic worlds. The Planck speed is always zero. This is fully possible without contradicting the relativity principle because the Planck mass particle has a radius equal to the Planck length and only lasts the Planck time before dissolving into photons. The Planck mass particle is two colliding photons. So, this collision can only be observed directly from its own reference frame, and therefore it always stands still relative to the direct observer. However, we can indirectly observe it as gravity from other frames. The missing Planck speed, and understanding how it is related to mass, is part of the reason why we not have been able to unify gravity with quantum mechanics in a simple and robust way until recently [13], [14]. We have also looked at the upper speed limit $c_{g}$ from a gravity perspective and demonstrated that $\frac{l_{p}}{t_{p}}$ can easily be extracted from easily-observable gravity phenomena without any knowledge of $G$, $\hbar$, and $c$. Actually, we can say the most important speed (the Planck speed) is binary, it is zero and $c$, zero when colliding and $c_{g}=c$ when the indivisible particles not are colliding. 


\section{APPENDIX}

To come up with the formula for the Planck length from time dilation we start out with standard gravitational time dilation

$$
T_{h}=T_{L} \frac{\sqrt{1-\frac{v_{e, h}^{2}}{c^{2}}}}{\sqrt{1-\frac{v_{e, L}^{2}}{c^{2}}}}=T_{L} \frac{\sqrt{1-\frac{2 G M}{R_{h} c^{2}}}}{\sqrt{1-\frac{2 G M}{R_{L} c^{2}}}}
$$

This can actually also be calculated from Newton gravity as the escape velocity is the same there as in general relativity theory. Further we have that we can solve the Planck length formula, $l_{p}=\sqrt{\frac{G \hbar}{c^{3}}}$, with respect to $G$, this gives

$$
G=\frac{l_{p}^{2} c^{3}}{\hbar}
$$

That is we will claim the Newton gravitational constant is a composite constant of this form, see [48]. Next we have that the standard escape velocity is given by

$$
v_{e}=\sqrt{\frac{2 G M}{R}}=\sqrt{\frac{2 \frac{l_{p}^{2} c^{3}}{\hbar} \frac{\hbar}{\lambda_{M}} \frac{1}{c}}{R}}=c l_{p} \sqrt{\frac{2}{R \bar{\lambda}_{M}}}
$$

This can be found from both Newton gravity and general relativity theory [61], [62], as the escape velocity is the same in the two models.

Replacing this into the time dilation formula we get

$$
T_{h}=T_{L} \frac{\sqrt{1-\frac{2 l_{p}^{2}}{\lambda_{M} R_{h}}}}{\sqrt{1-\frac{2 l_{p}^{2}}{\lambda_{M} R_{L}}}}
$$

Next solving this with respect to $l_{p}$ we get

$$
l_{p}=\frac{\sqrt{\bar{\lambda}_{M} R_{L} R_{h}\left(T_{h}^{2}-T_{L}^{2}\right)}}{\sqrt{2\left(R_{h} T_{h}^{2}-R_{L} T_{L}^{2}\right)}}
$$

A formula was first presented in [10]. Similar the other formulas can be derived. The break through in derivations of the Planck length independent of knowledge of $G, c$ and $\hbar$ comes from understanding that $G$ is a composite constant of the form given, and that all masses in kilogram can be represented simply by solving the reduced Compton wavelength formula $\bar{\lambda}=\frac{\hbar}{m c}$ with respect to $m$. Further that the Planck constant also cancels out when one then multiply the composite form of $G$ with the mass described as $M=\frac{\hbar}{\lambda_{M}} \frac{1}{c}$, where we use the notation $\bar{\lambda}_{M}$ for the reduced Compton wavelength, simply to show that it is the large mass $M$ in the Newton formula (or GR) we are working with here.

\section{REFERENCES}

[1] M. Planck. Natuerliche Masseinheiten. Der Königlich Preussischen Akademie Der Wissenschaften, 1899.

[2] A. S. Eddington. Report On The Relativity Theory Of Gravitation. The Physical Society Of London, Fleetway Press, London, 1918.

[3] T. Padmanabhan. Planck length as the lower bound to all physical length scales. General Relativity and Gravitation, 17 , 1985. https://doi.org/10.1007/BF00760244.

[4] R. J. Adler. Six easy roads to the Planck scale. American Journal of Physics, 78(9):925, 2010. URL https://doi.org/10.1119/1.3439650.

[5] S. Hossenfelder. Can we measure structures to a precision better than the Planck length? Classical and Quantum Gravity, 29, 2012. URL https://doi.org/10.1088/0264-9381/29/11/115011.

[6] S. Hossenfelder. Minimal length scale scenarios for quantum gravity. Living Reviews in Relativity, 16, 2013. URL https://doi.org/10.12942/lrr-2013-2.

[7] A. Unzicker. The Mathematical Reality: Why Space and Time Are an Illusion. Independently published, 2020.

[8] E. G. Haug. Can the Planck length be found independent of big $G$ ? Applied Physics Research, 9(6):58, 2017. URL https://doi.org/10.5539/apr.v9n6p58.

[9] E. G. Haug. Finding the Planck length multiplied by the speed of light without any knowledge of $G$, $c$, or $h$, using a Newton force spring. Journal Physics Communication, 4:075001, 2020a. URL https://doi.org/10.1088/2399-6528/ab9dd7.

[10] E. G. Haug. Demonstration that Newtonian gravity moves at the speed of light and not instantaneously (infinite speed) as thought! Journal of Physics Communication., 5(2):1, 2021a. URL https://doi.org/10.1088/2399-6528/abe4c8.

[11] E. G. Haug. Using a grandfather pendulum clock to measure the world's shortest time interval, the Planck time (with zero knowledge of $G$ ). Journal of Applied Mathematics and Physics, 9:1076, 2021b.

[12] E. G. Haug. Measurements of the Planck length from a ball-clock without knowledge of Newton's gravitational constant $G$ or the Planck constant. European Journal of Applied Physics, 3:15, 2021c.

[13] E. G. Haug. Collision space-time: Unified quantum gravity. Physics Essays, 33(1):46, 2020b. URL https://doi.org/10.4006/0836-1398-33.1.46.

[14] E. G. Haug. Rethinking the foundation of physics and its relation to quantum gravity and quantum probabilities: Unification of gravity and quantum mechanics. Preprints.org, 2020c. URL https://www.preprints.org/manuscript/202012.0483/v2.

[15] E. G. Haug. Quantum Gravity Hidden In Newton Gravity And How To Unify It With Quantum Mechanics. in the book: The Origin of Gravity from the First Principles, Editor Volodymyr Krasnoholovets, NOVA Publishing, New York, 2021d.

[16] J. R. Buczyna, C. S. Unnikrishnan, and G. T. Gillies. Standard and derived Planck quantities: Selected analysis and observations. Soviet Astronomy Letters, 17(4), 1978.

[17] E. R. Caianiello. Is there a maximal acceleration? Letter Nuovo Cimento, 32:65, 1981. URL https://doi.org/10.1007/BF02745135.

[18] G. Scarpetta. Letter Nuovo Cimento, 51, 1984.

[19] E. R Caianiello, S. D. Filippo, G. Marco, and Vilasi. G. Remarks on the maxlmal-acceleration hypothesis. Letter Nuovo Cimento, $34: 112$, 1982. 
[20] E. R. Caianiello and Landi G. Maximal acceleration and Sakharov's limiting temperature.

[21] D. F. Falla and P. T. Landsberg. Black holes and limits on some physical quantities. European Journal of Physics, $15,1994$.

[22] J. R. Buczyna, C. S. Unnikrishnan, and G. T. Gillies. Standard and derived Planck quantities: selected analysis and derivations. Gravitation and Cosmology, 17(4):339, 2011. URL https://doi.org/10.1134/S0202289311040037.

[23] H. Poincaré. The measure of time. English translation by George Bruce Halsted 1913 in the Foundation of Science, 1898.

[24] Georges Sagnac. The demonstration of the luminiferous aether by an interferometer in uniform rotation. Comptes Rendus, 157:708-710, 1913.

[25] G. Spavieri and E. G. Haug. Why the Sagnac effect favors absolute over relative simultaneity. Physics Essays, 32(2):331, 2019. URL https://doi.org/10.4006/0836-1398-32.3.331.

[26] G. Spavieri, G. Gillies, G. Haug, and A. Sanchez. Light propagation and local speed in the linear Sagnac effect. Journal of Modern Optics, 26(21): 2131, 2019. URL https://doi.org/10.1080/09500340.2019.1695005.

[27] E. T Kipreos and R. S Balachandran. Assessment of the relativistic rotational transformations. Modern Physics Letters A, 36:2150113, 2021. URL https://doi.org/10.1142/S0217732321501133.

[28] B. P. Abbott et. al. Gravitational waves and gamma-rays from a binary neutron star merger: Gw170817 and GRB 170817a. The Astrophysical Journal Letters, Volume 848, 848(2):1?27, 2017. URL https://doi.org/0000-0002-7537-3210.

[29] W. W. Salisbury. Velocity of gravitational waves. Nature, 224, 1969. URL https://doi.org/10.1038/224782a0.

[30] S. Du, F. Peng, and M. Li. Measuring the speed of gravitational waves with the distorted pulsars. arXiv:1712.05965, 2017.

[31] N. Cornish, D. Blas, and G. Nardini. Bounding the speed of gravity with gravitational wave observations. Physics Review Letter, 119, 2017. URL https://doi.org/10.1103/PhysRevLett.119.161102.

[32] E. G. Haug. Extraction of the speed of gravity (light) from gravity observations only. International Journal of Astronomy and Astrophysics, 9(2), 2019. URL https://doi.org/10.4236/ijaa.2019.92008.

[33] O. J. Pike, F. Mackenroth, E. G. Hill, and Rose S. J. A photon-photon collider in a vacuum Hohlraum. Nature Photonics, 8, 2014. URL https://doi.org/10.1038/nphoton.2014.95.

[34] E. G. Haug. Newton and Einstein's gravity in a new perspective for Planck masses and smaller sized objects. International Journal of Astronomy and Astrophysics, 8, 2018. URL https://doi.org/10.4236/ijaa.2018.81002.

[35] A. H. Compton. A quantum theory of the scattering of x-rays by light elements. Physical Review, 21(5):483, 1923. URL https://doi.org/10.1103/PhysRev.21.483.

[36] H. A. Lorentz. Simplified theory of electrical and optical phenomena in moving systems. Proc. Acad. Scientific, Amsterdam, 1, 1899.

[37] A. Einstein. On the electrodynamics of moving bodies. Annalen der Physik, English translation by George Barker Jeffery 1923, (17), 1905. URL https://doi.org/10.1002/andp.19053221004.

[38] D. C Giancoli. Physics for Scientists \& Engineers. Pearson Prentice Hall, New Jersey, 2009.

[39] P. A. Tipler and R. A. Llewellyn. Modern Physics, Third Edition. W. H. Freeman and Company, New York, 1999.

[40] H. Minikowski's wski. Space and time. A Translation of an Address delivered at the 80th Assembly of German Natural Scientists and Physicians, at Cologne, 21 September, in the book "The Principle of Relativity", Dover 1923, 1908.

[41] E. Hecht. Einstein never approved the relativistic mass formula. The Physics Teacher, 47:336, 2009. URL https://doi.org/10.1119/1.3204111.

[42] E. G. Haug. Three dimensional space-time gravitational metric, 3 space +3 time dimensions. Journal of High Energy Physics, Gravitation and Cosmology, 7:1230, 2021e. URL https://doi.org/10.4236/jhepgc.2021.74074.

[43] E. G. Haug. A new full relativistic escape velocity and a new Hubble related equation for the universe. Physics Essays, 34(4):502, 2021f. URL http://dx.doi.org/10.4006/0836-1398-34.4.502.

[44] M. Planck. Das prinzip der relativität und die grundgleichungen der mechanik. Verhandlungen Deutsche Physikalische Gesellschaft, 4, 1906.

[45] C. G. Adler. Dose mass really depends on velocity dad? American Journal of Physics, 55:739, 1987. URL https://doi.org/10.1119/1.15314.

[46] E. G. Haug. Relativistic Newtonian gravity makes dark energy superfluous? https://vixra.org/abs/2004.0041, 2020d.

[47] E. G. Haug. Wormholes do not exist, they are mathematical artifacts from an incomplete gravitational theory (?). HAL archive, 2021g. URL https://hal.archives-ouvertes.fr/hal-03325115/document.

[48] E. G. Haug. The gravitational constant and the Planck units. A simplification of the quantum realm. Physics Essays, 29(4):558, 2016. URL https://doi.org/10.4006/0836-1398-29.4.558.

[49] E. G. Haug. The ultimate limits of the relativistic rocket equation. The Planck photon rocket. Acta Astronautica, 136, 2017b. URL https://doi.org/10.1016/j.actaastro.2017.03.011.

[50] E. G. Haug. Unified Revolution, New Fundamental Physics. Oslo, E.G.H. Publishing, 2014.

[51] E. G. Haug. Does special relativity lead to a Trans-Planckian crisis? Applied Physics Research, 12(1), 2020e. URL https://doi.org/10.5539/apr.v12n1p1.

[52] E. Schrödinger. Über die kräftefreie bewegung in der relativistischen quantenmechanik. Sitzungsberichte der Preußischen Akademie der Wissenschaften. Physikalisch-mathematische Klasse, 1930.

[53] L.S. Levitt. The proton Compton wavelength as the 'quantum' of length. Experientia, 14:233, 1958. URL https://doi.org/10.1007/BF02159173.

[54] O. L. Trinhammer and H. G. Bohr. On proton charge radius definition. EPL, 128:21001, 2019. URL https://doi.org/10.1209/0295-5075/128/21001.

[55] G.T. Gillies, Luo J., and L. C. Tu. The mass of the photon. Reports on Progress in Physics, 6, 2005.

[56] G. Spavieri, J. Quintero, G.T. Gilles, and Rodriguez M. A survey of existing and proposed classical and quantum approaches to the photon mass. The European Physical Journal D, 61:1, 2011. URL https://doi.org/10.1140/epjd/e2011-10508-7.

[57] J. Christian. Passage of time in a Planck scale rooted local inertial structure. arXiv:gr-qc/0308028 v3, 2003. URL arXiv:gr-qc/0308028 v3.

[58] J. P. Bruneton. Notes on several phenomenological laws of quantum gravity. arXiv, 2013. URL https://arxiv.org/pdf/1308.4044v1.pdf.

[59] D. Meschini. Planck-scale physics: Facts and beliefs. Foundations of Science, 12:277, 2007. URL https://doi.org/10.1007/s10699-006-9102-3.

[60] D. Mermin. Space and Time in Special Relativity. Waveland Pr Inc, 1989.

[61] Bernard Schutz. Gravity from the Ground Up. Cambridge University Press, 2003.

[62] A. T. Augousti and A. Radosz. An observation on the congruence of the escape velocity in classical mechanics and general relativity in a Schwarzschild metric. European Journal of Physics, 376:331-335, 2006. URL https://doi.org/10.1088/0143-0807/27/2/015. 